\title{
Application of gene therapy in the treatment of superficial digital flexor tendon injury in horses
}

\author{
Alexandr Aimaletdinov ${ }^{1}$, Gulnur Mindubaeva ${ }^{2}$, Svetlana Khalikova ${ }^{2}$, Emmanuel Kabwe $^{1,3}$, Alexandra Salmakova ${ }^{1}$, \\ Natalia Alexandrova ${ }^{1}$, Catrin Rutland ${ }^{4}$, Albert Rizvanov ${ }^{1}$ and Elena Zakirova ${ }^{1 *}$ \\ ${ }^{1}$ Department of Exploratory Research, Scientific and Educational Center of Pharmaceutics, Institute of \\ Fundamental Medicine and Biology, Kazan Federal University, Kazan, Russian Federation \\ ${ }^{2}$ International equestrian center "Kazan», Russian Federation \\ ${ }^{3}$ Kazan Research Institute of Epidemiology and Microbiology, Kazan, the Republic of Tatarstan, Russian Federation \\ ${ }^{4}$ Faculty of Medicine, School of Veterinary Medicine and Science, University of Nottingham, Nottingham, United \\ Kingdom
}

\begin{abstract}
Background: Tendon injuries are one of the most common causes of orthopedic disorders in horses. Such injuries involve a long course of treatment and recovery. The most promising method of treating these injuries is the use of recombinant proteins and gene therapy.

Aim: In this work, we evaluated the therapeutic efficacy of plasmid DNA (pDNA) containing two species-specific coding sequences, i.e. vascular endothelial growth factor 164 (VEGF164) and fibroblast growth factor 2 (FGF2), in the treatment of severe damage to the tendon of the superficial digital flexor.

Methods: A pDNA construct was used to restore the damaged superficial digital flexor tendon in the horse.

Results: This study showed that the administration of pDNA encoding VEGF164 and FGF2 genes at the injury area increased the regenerative activities of the damaged tendon.

Conclusion: This study shows the therapeutic properties of genetic constructs (pDNA) and contributes to the advancements in the use of these therapies.

Keywords: Fibroblast growth factor 2, Plasmid DNA, Superficial digital flexor, Vascular endothelial growth factor.
\end{abstract}

\section{Introduction}

Tendon or ligament injuries are the common causes of orthopedic disorders in active horses (Equus ferus caballus) of any age and breed (Thorpe et al., 2010). According to statistics, injuries amount to $86 \%$ of all diseases, whereas $37 \%$ are due to the pathology of muscles, tendons, and joints in sports horses. These injuries may involve a long recovery course, which can last up to 9-12 months (O’Meara et al., 2010). Injuries to the tendon-ligamentous apparatus cause the horses to lose their working capacity and are released from work for as long as they do not recover. Moreover, the injuries lead to chronic diseases in the musculoskeletal system, which are manifested by the destructivedystrophic damage to collagen fibers of tendons, as well as adjacent and underlying tissues (Nogteva et al., 2012; Vlasov et al., 2015). Most sick horses (up to $80 \%$ ) with micro- and macro-tendon ruptures suffered from repeated injuries after 3-12 months following their initial injuries (Dowling et al., 2000).

The main causes of horse tendon injuries are: sudden tendon extension with a sharp contraction of muscles, an early high level of physical activities, and tendon hyperthermia (Dyson, 2004; Kovac, 2013). Tendonitis is characterized by microtrauma, rupture of tendon fibers and fibrils, and intracranial microcirculation, leading to the death of tenocytes and the development of aseptic inflammatory processes (Dahlgren, 2005; Smith et al., 2014). The long healing process occurs due to hypovascularity in combination with tendon hypocellularity. Scarring after damage to the tendons can cause a rupture in the tendons of a predisposed horse and may occur due to increased expression of collagen type III (COL3), which has less fiber and less crosslinking compared to collagen type I (COL1), which leads to a deterioration in its mechanical properties (Patterson-Kane et al., 2012; O’Brien and Smith, 2018).

Currently, recombinant proteins and gene therapy are the most emerging advanced therapeutic methods used in the treatment of the different musculoskeletal problems in both human (Martinek et al., 2005) and veterinary medicines. The aim of this study is to evaluate the therapeutic efficacy of gene therapy plasmid DNA (pDNA) containing species-specific vascular endothelial growth factor (VEGF164) and fibroblast growth factor 2 (FGF2) coding sequences for the treatment of severely damaged superficial digital flexor tendons of the horse.

\footnotetext{
*Corresponding Author: Elena Zakirova. Department of Exploratory Research, Scientific and Educational Center of Pharmaceutics, Institute of Fundamental Medicine and Biology, Kazan Federal University, Kazan, Russian Federation.
} 


\section{Materials and Methods}

\section{Plasmid DNA}

In this study, we used pDNA containing coding sequences of species-specific VEGF164 and FGF2 genes used in our previous case report (Litvin et al., 2016). The pDNA in this study was a genetic construct (named pBUDK-ecVEGF164-ecFGF2) developed to repair and restore damaged connective tissues in tendons and ligaments. The recombinant plasmid contained one of the coding sequences from the VEGF and FGF proteins growth factor families ( $E$. caballus VEGF164 and FGF2, respectively). Also, the use of the construct pBUDK-ecVEGF164-ecFGF2 pDNA was reported previously (Litvin et al., 2016). The dosage of the pDNA was checked on the agarose gel electrophoresis, the resultant sterile drug contained $\geq 95 \%$ pDNA in a supercoiled form and the endotoxin content was $\leq 0.03 \mathrm{EU} / \mathrm{mg}$ pDNA in lyophilized form (aliquoted in doses of $5 \mathrm{mg}$ ).

\section{Preparation and introduction of plasmid DNA}

Before the introduction of pDNA into the damaged ligaments, the liquid pDNA was warmed to $+37^{\circ} \mathrm{C}$. Then, $3.5 \mathrm{ml}$ was injected directly into the damaged sites following the ultrasonic and aseptic conditions guidelines. Multi-injections were administered into the affected sites at a distance of $1 \mathrm{~cm}$ apart to ensure a uniformed and guaranteed distribution of the drug throughout the damaged tissues. For the entire treatment, just a single injection of the drug (pDNA) was used. Following drug administration, the wounded area was tightly covered with bandages and the horse was given strict rest for 2 days, and then step load exercise rehabilitation was prescribed for 3 weeks.

\section{Ethical approval}

All human standard guidelines established by the US Food and Drug Administration for safe administration
(Food and Drug Administration, 2007; Content and Review of Chemistry, Manufacturing, and Control (CMC) Information for Human Somatic Cell Therapy Investigational New Drug Applications, 2008) and the Human Medicines Committee of the European Medicines Agency (European Medicines Agency, 2011, 2015) were adhered to when designing the plasmid vectors. For the treatment phase, a horse with a naturally occurring injury was brought to a veterinary clinic at Kazan Hippodrome (an international equestrian center). Informed consent to administer a therapeutic drug was obtained from the owners. All procedures were carried out in accordance with the international, national, and institutional guidelines for the care and use of animals. The Institutional Review Board of the Kazan Federal University approved this study (under protocol No3; date 05/05/2015).

\section{Results}

Therapeutic efficacy and the regenerative effect of the drug (pDNA) on connective tissues were studied in a 2-year-old pure-bred Arabian stallion with damage to the superficial extensor. During clinical examination, it was observed that the horse was limping on the left forelimb. The degree of lameness was rated 2 out of 5 (according to the 5-point lameness scale (2) AAEP). The pronounced anechoic and hypoechoic zones at the back of the limb was visualized using ultrasound examination of the damaged leg (Fig. 1). A diagnosis of tendonitis of the superficial digital flexor was made.

On the 10th day after pDNA administration, clinical examination confirmed no lameness on the previously affected left forelimb. An ultrasound examination of the superficial digital flexor revealed increased echogenicity in the damaged area, indicating heterogeneity of the

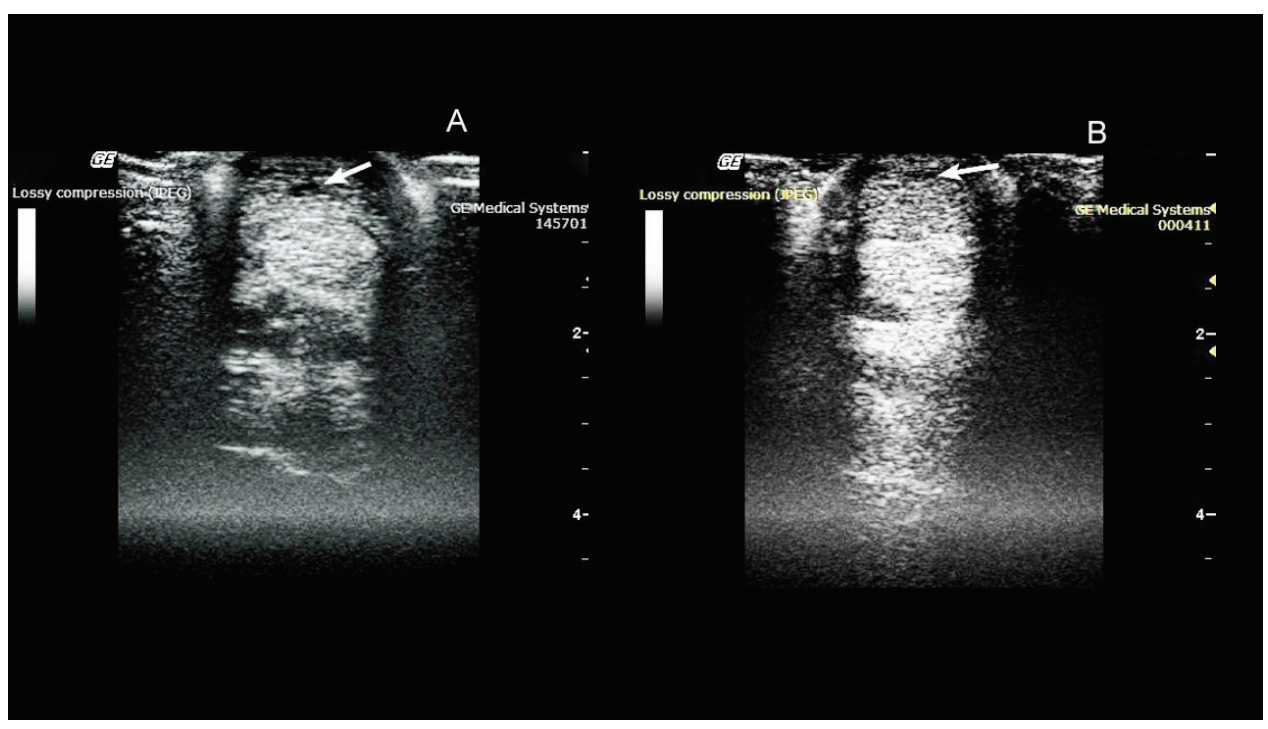

Fig. 1. Ultrasonography of the superficial digital flexor. (A): Before the introduction of plasmid DNA. (B): 20th day after the introduction of plasmid DNA. 


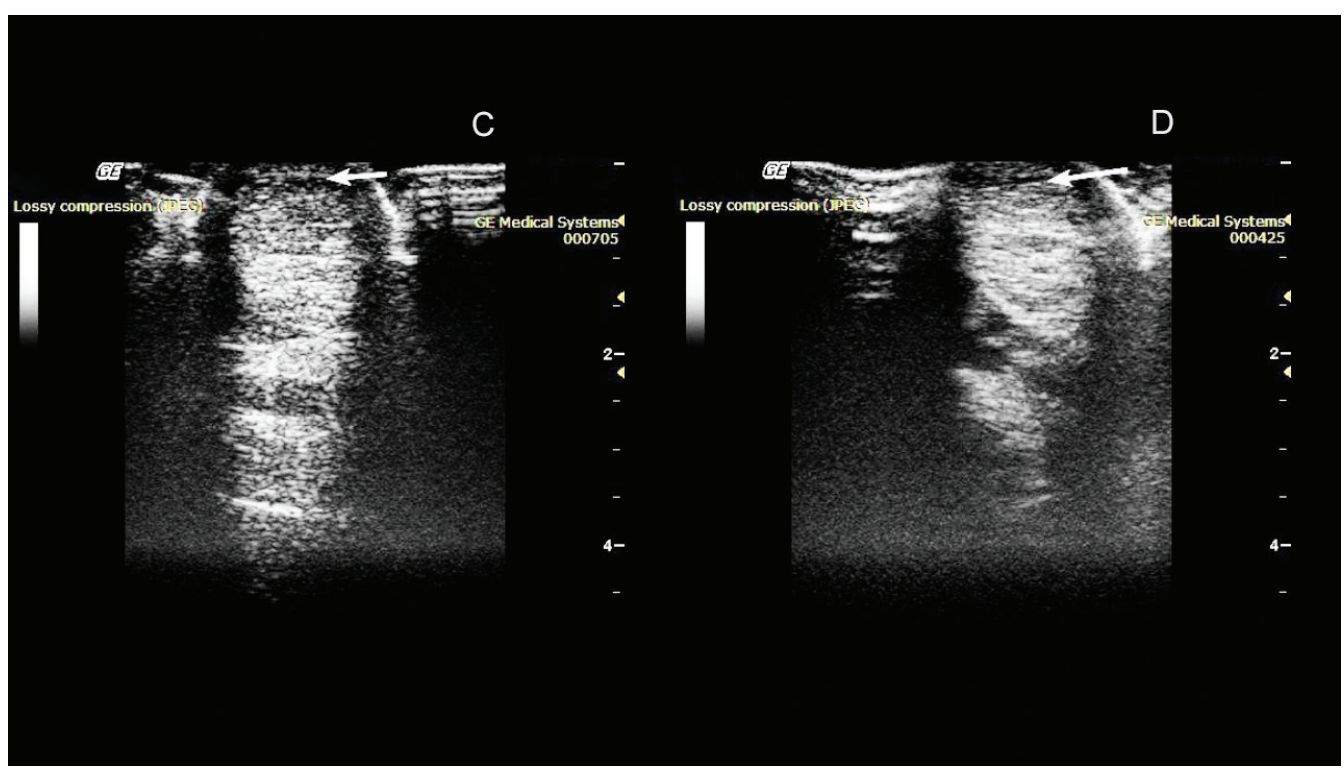

Fig. 2. Ultrasonography of the superficial digital flexor. (C): 50th day after the introduction of plasmid DNA. (D): 80th day after the introduction of plasmid DNA.

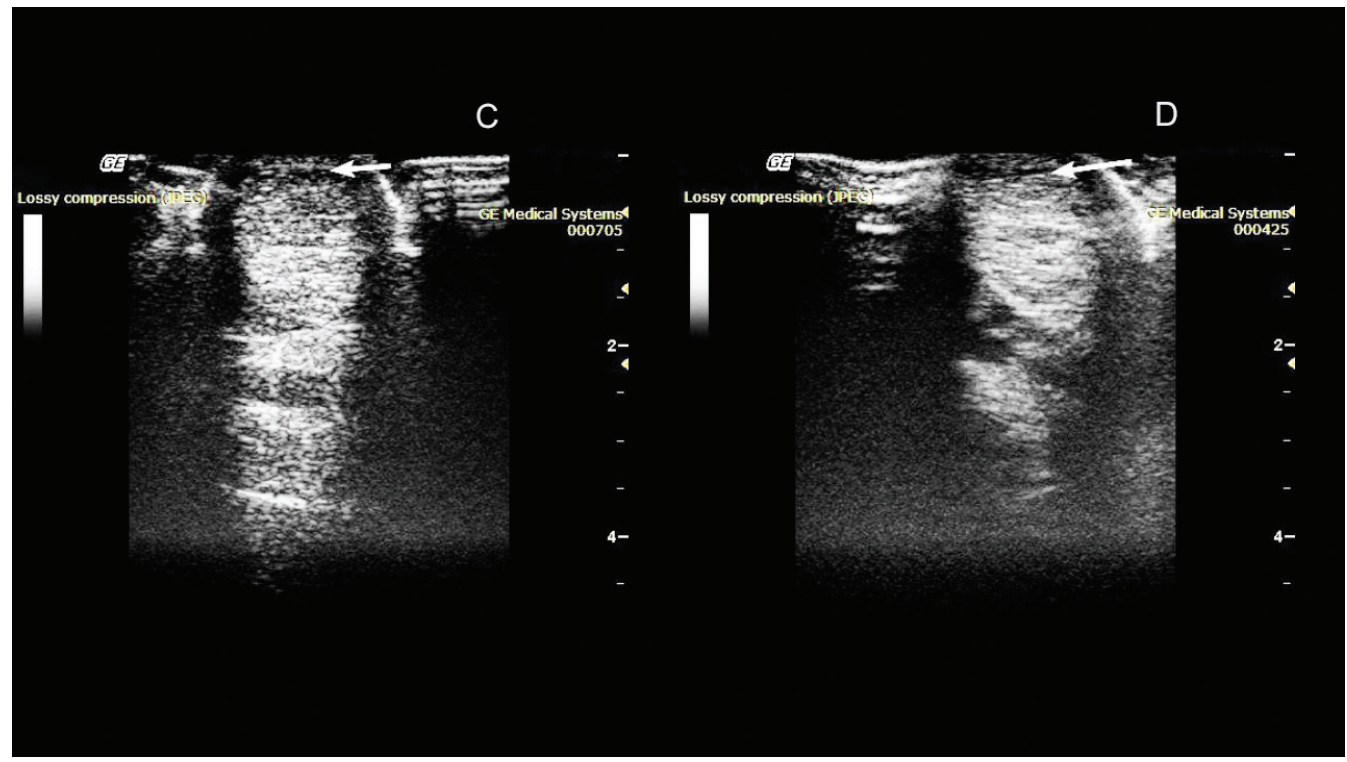

Fig. 3. Ultrasonography of the superficial digital flexor. (E): 110th day after the introduction of plasmid DNA. (F): 140th day after the introduction of plasmid DNA.

structure in the damaged area, due to the formation of connective tissue.

On the 20th day after drug administration, the absence of lameness and lack of swelling at the site of damage were observed. An ultrasound scan showed an increase in the degree of echogenicity and a decrease in the hypoechoic region of the damaged area (Fig. 1).

On the 50th day after administration of the drug, the absence of lameness was noted during the examination. Ultrasound examination showed a decrease in the hypoechoic zone, and an increase in echogenicity at the site of damage was also observed (Fig. 2).

On the 80th day, the absence of lameness was still observed. Ultrasound examination showed a decrease in the hypoechoic zone and an increase in echogenicity at the site of injury. This indicated the presence of a reparative process. As the clinical and ultrasound characteristics of the damaged tendon improved, a gradual introduction of trotting was prescribed in addition to walking. 
On the 110th day, after administration of the drug, the horse was examined again in the clinic. The examination showed the absence of lameness in the left thoracic limb when the horse was moving on hard and soft ground, during both walking steps and trotting. Ultrasound examination revealed that the structure of the superficial digital flexor was close to normal tendon tissue. The tendon structure was quite uniform throughout its length, and the sites of partial damage to the ligament were hardly seen.

On the 140th day, a decrease in the hypoechoic zone was observed during ultrasound examination. There was no swelling at the site of injury and clinical examination showed the absence of claudication. Also, the horse calmly rested on the injured leg. Furthermore, clinical data, as well as ultrasound examination, revealed the presence of regeneration processes on this day (Fig. 3). On the 185th day, after clinical examination, deterioration of the damaged limb was observed. Ultrasound examination revealed no changes in the area of drug administration. However, a new lesion was discovered in this tendon.

On the 210th day, ultrasound revealed a significant increase in the hypoechoic zone in the new lesion. It also showed the presence of damage to the deep digital flexor tendon. Upon external examination, slight swelling and lameness were noted.

No adverse reactions to the drug or contaminations at the site of infection were noted at any point during the treatment process.

\section{Discussion}

In dressage horses, the most common injuries occur in the digital flexor tendon and suspensory ligament due to extensive training and sporting activities causing orthopedic disorders. As a result, the performances of the horses are reduced which can lead to early retirement from competition, and in most cases, the traditional medications and regimes used to treat them still result in relapses of the injuries in later years. One of the significant problems with orthopedic disorders is the prevention of blood supply to the ischemic tissues in the injury area. Currently, one of the most promising approaches to the restoration of blood supply to ischemic tissues is gene therapy aimed at ensuring the growth of new blood vessels. Evidently, angiogenesis begins with the migration of endothelial cells into the intercellular space in the direction of the occluded vessel. The main molecular factors initiating and controlling this process are, to a greater extent, various types of VEGFs, and to a lesser extent, FGFs (Sylven, 2002). Thus, the transfer of genes of these factors into ischemic tissues can initiate local growth and development of blood vessels by overexpression of specific proteins. To make the gene therapy drug, we used the species-specific VEGF164 and FGF2 genes. These genes encode proteins that promote the process of regeneration of both blood vessels and connective tissues in the equine. Also, VEGF164 in vivo stimulates angiogenesis by attracting endothelial progenitor cells from the bone marrow. In addition, it stabilizes newly formed vessels and increases their permeability at the site of damage, which contributes to the formation of granulation tissues (Litvin et al., 2016). Correspondingly, FGF2 induces proliferation of cells, and regeneration of the nervous, muscular, and connective tissues. Growth factors VEGF and FGF2 are known to be polypeptides that are mainly released by platelets, fibroblasts, and endothelial cells at the site of injury (Byrne et al., 2005). They are closely related to other factors of inflammation and regeneration. It is believed that they stimulate the migration of tendoblasts, fibroblasts, and mesenchymal stem cells, which are responsible for the production of collagen and other extracellular components of ligaments and tendons, such as proteoglycans, glycosaminoglycans, and glycoproteins, through enhanced angiogenesis (the process of formation of new vessels) at the site of damage (Martinek et al., 2005).

The in vivo efficacy of pBUDK-ecVEGF164-ecFGF pDNA was confirmed previously by us in the treatment of tendon injury of the superficial digital flexor (Kovac et al., 2017). Treatment with gene therapy led to a significant improvement in the condition of the animal. Three months after the introduction of pDNA encoding VEGF and FGF2 genes, the damaged tendon regenerated. Dynamic ultrasound examinations at the damaged tendon area of the superficial digital flexor showed a significant decrease in the hypoechoic region. During this period, the horse was released for training and it was planned that the animal would be introduced into normal sports mode load after three months. However, two months after the start of training, the animal began to limp and new but separate damage to the tendon of the superficial digital flexor was discovered. According to an ultrasound scan, the new lesion was located above the area that had been successfully restored using gene therapy. We believe that damage to the intact tendon during training occurred as a result of microtrauma received as a result of past competitions and not due to the recovery after the gene therapy. This suggests that gene therapy treatment has a pronounced therapeutic effect with high efficiency. However, to confirm this hypothesis, a histological and immunohistochemical study of biopsy materials obtained from a damaged tendon is required. It is also necessary to be familiar with the causes of relapses in tendon injuries when applying gene therapy methods.

Currently, gene therapy is the most promising method of treatment in regenerative medicine. This opens up wide possibilities for its use in the treatment of orthopedic pathology, in particular for the treatment of damaged ligaments and tendons (Martinek et al., 2005; Evans et al., 2009; Tang et al., 2016).

Gene therapy as a novel method for the treatment of orthopedic disorders has been actively used not only 
in horses (Kovac et al., 2017; 2018), but also in other animals and humans (Plotnikov et al., 2012; Zakirova et al., 2014). The application of a construct plasmid encoding the VEGF164 and BMP2 genes to treat the damage to the anterior cruciate ligament in a dog was previously described (Kovac et al., 2018). Also, gene therapy was used for the treatment of lower limb ischemia in humans. In all these treatments, the use of genetic contracts has proved efficiency and safety (Plotnikov et al., 2012; Deev et al., 2015; 2017). Thus, this study shows that the introduction of pDNA encoding specie-specific VEGF164 and FGF2 genes increased the regenerative activities of the damaged tendon. However, it is necessary to be more careful about horse training, since the possibility of damage to other parts of the treated tendon cannot be ruled out. Although there are many challenges in the use of gene therapy, this study shows the therapeutic properties of genetic constructs (pDNA) and contribute to the advancements in the use of these therapies.

\section{Acknowledgments}

This study was funded by the Russian Foundation for Basic Research (RFBR), project number 20-016-00022 and Kazan Federal University was supported by the Russian Government Program of Competitive Growth. Authors' contributions

Conceptualization: Aimaletdinov Alexandr, Mindubaeva Gulnur and Khalikova Svetlana. Investigation: Aimaletdinov Alexandr and Zakirova Elena. Formal analysis: Salmakova Alexandra and Rutland Catrin. Visualization: Alexandrova Natalia and Kabwe Emmanuel. Funding acquisition: Rizvanov Albert and Zakirova Elena. Supervision: Rizvanov Albert and Zakirova Elena. Writing the original draft: Aimaletdinov Alexandr and Zakirova Elena. Final editing: Aimaletdinov Alexandr, Mindubaeva Gulnur, Khalikova Svetlana, Kabwe Emmanuel, Salmakova Alexandra, Alexandrova Natalia, Rutland Catrin, Rizvanov Albert, and Zakirova Elena.

\section{Conflict of interest}

The authors declare that there is no conflict of interest.

\section{References}

Byrne, A.M., Bouchier-Hayes, D.J. and Harmey, J.H. 2005. Angiogenic and cell survival functions of vascular endothelial growth factor (VEGF). J. Cell Mol. Med. 9(4), 777-794.

Content and Review of Chemistry, Manufacturing, and Control (CMC) Information for Human Somatic Cell Therapy Investigational New Drug Applications (INDs). 2008. U.S. Department of Health and Human Services, Food and Drug Administration, Center for Biologics Evaluation and Research. April 2008, Available via http:// www.fda.gov/cber/guidelines.htmDahlgren, L.A. 2005. Cloning and expression of type III collagen in normal and injured tendons of horses. Am. J. Vet. Res. 66, 266-270.

Deev, R., Plaksa, I., Bozo, I. and Isaev, A. 2017. Results of an international postmarketing surveillance study of pl-VEGF165 safety and efficacy in 210 patients with peripheral arterial disease. Am. J. Cardiovasc. Drugs 17(3), 235-242.

Deev, R.V., Bozo, I.Y., Mzhavanadze, N.D., Voronov, D.A., Gavrilenko, A.V., Chervyakov, Y.V., Staroverov, I.N., Kalinin, R.E., Shvalb, P.G. and Isaev, A.A. 2015. pCMV-vegf165 intramuscular gene transfer is an effective method of treatment for patients with chronic lower limb ischemia. J. Cardiovasc. Pharmacol. Ther. 20(5), 473-482.

Dowling, B.A., Dart, A.J., Hodgson, D.R. and Smith, R.K.W. 2000. Superficial digital flexor tendonitis in the horse. Equine Vet. J. 32, 369-378.

Dyson, S.J. 2004. Medical management of superficial digital flexor tendonitis: a comparative study in 219 horses (1992-2000). Equine Vet. J. 36, 415-419.

European Medicines Agency. 2015. Guideline on the non-clinical studies required before first clinical use of gene therapy medicinal products. 23 March 2015, EMA/CAT/80183/2014, Committee for Advanced Therapies (CAT). London, UK: European Medicines Agency. EMEA/CHMP/ GTWP/125459/2006.

European Medicines Agency. 2011. Reflection paper on design modifications of gene therapy medicinal products during development. London, UK: European Medicines Agency. 14 December 2011, EMA/CAT/GTWP/44236/2009, Committee for advanced therapies.

Evans, C.H., Ghivizzani, S.C. and Robbins, P.D. 2009. Orthopedic gene therapy in 2008. Mol. Ther. 17(2), 231-244.

Food and Drug Administration (FDA). 2007. Considerations for plasmid DNA vaccines for infectious disease indications; industry guidance, 8378-8379 [05-3106]. Food and Drug Administration, November 2007. Available via http://www.fda.gov/cber/guidelines.htm

Kovac, M. 2013. Orthopedic diseases of horsesmodern methods of diagnosis and treatment. In 2nd ed. Ed., Volcova E. Royal Publishing House, Moscow, Russian Federation, pp: 635.

Kovac, M., Litvin, Y.A., Aliev, R.O., Zakirova, E.Y., Rutland, C.S., Kiyasov, A.P. and Rizvanov, A.A. 2017. Gene therapy using plasmid DNA encoding vascular endothelial growth factor 164 and fibroblast growth factor 2 genes for the treatment of horse tendinitis and desmitis. Case reports. Front. Vet. Sci. 4, 1-6.

Kovac, M., Litvin, Y.A., Aliev, R.O., Zakirova, E.Y., Rutland, C.S., Kiyasov, A.P. and Rizvanov, A.A. 2018. Gene Therapy Using Plasmid DNA Encoding VEGF164 and FGF2 Genes: A Novel Treatment of Naturally Occurring Tendinitis and Desmitis in 
Horses. Front. Pharmacol. 9, 978; doi: 10.3389/ fphar.2018.00978.

Litvin, Y.A., Zakirova, E.Y., Zhuravleva, M.N. and Rizvanov, A.A. 2016. Generation of plasmid DNA expressing species-specific horse VEGF164 and FGF2 factors for gene therapy. BioNanoSci. 6, 550-553.

Martinek, V., Huard, J. and Fu, F.H. 2005. Gene therapy in tendon ailments. In Tendon Injuries, $(\mathrm{N}$. Maffulli, P. Renström, and W. B. Leadbetter eds). London, Springer, pp: 307-312.

Nogteva, I.V., Popryaduhina, P.V., Petrova, N.A., Romanova, O.V. and Smirnova, N.V. 2012. Modern methods of regenerative medicine in traumatology and orthopedics of horses. Hippol. Vet. Med. 3(5), $43-50$.

O'Meara, B., Bladon, B., Parkin, T.D., Fraser, B. and Lischer, C.J. 2010. An investigation of the relationship between race performance and superficial digital flexor tendonitis in the Thoroughbred racehorse. Equine Vet. J. 42, 322326.

O'Brien, E.J.O. and Smith, R.K.W. 2018. Mineralization can be an incidental ultrasonographic finding in equine tendons and ligaments. Vet. Radiol. Ultrasound 59(5), 613-623.

Patterson-Kane, J.C., Becker, D.L. and Rich, T. 2012. The pathogenesis of tendon microdamage in athletes: the horse as a natural model for basic cellular research. J. Comp. Pathol. 147, 227-247.

Plotnikov, M.V., Rizvanov, A.A., Masgutov, R.F., Mavlikeev, M.O., Salafutdinov, I.I., Gazizov, I.M., Romanova, Y., Shamsutdinova, I., Bogov, A. and Maksimov, A. 2012. The first clinical experience of direct gene therapy using VEGF and bFGF in treatment patients with critical lower limb ischemia. Cell Transplant. Tissue Eng. 7(3), 180-184.

Smith, R., Mcllwraith, W., Schweitzer, R., Kadler, K., Cook, J., Caterson, B., Dakin, S., Heinegård, D., Screen, H., Stover, S., Crevier-Denoix, N., Clegg, P., Collins, M., Little, C., Frisbie, D., Kjaer, M., van Weeren, R., Werpy, N., Denoix, J.M., Carr, A., Goldberg, A., Bramlage, L., Smith, M. and Nixon, A. 2014. Advances in the understanding of tendinopathies: a report on the Second Havemeyer Workshop on equine tendon disease. Equine Vet. J. 46(1), 4-9.

Sylven, C. 2002. Angiogenic gene therapy. Drugs Today 38, 819-827.

Tang, J.B., Wu, Y.F., Cao, Y., Chen, C.H., Zhou, Y.L., Avanessian, B., Avanessian, B., Shimada, M., Wang, T.X. and Liu, P.Y. 2016. Basic FGF or VEGF gene therapy corrects insufficiency in the intrinsic healing capacity of tendons. Sci. Rep. 6, 20643; doi: $10.1038 /$ srep20643.

Thorpe, C.T., Clegg, P.D. and Birch, H.L. 2010. A review of tendon injury: why is the equine superficial digital flexor tendon most at risk? Equine Vet. J. 42, 174-180.

Vlasov, M.V., Bugrov, S.N., Bogos'Yan, A.B. and Musukhina, I.V. 2015. Experimental modeling of the processes of reparative regeneration of connective tissue in adverse conditions. Bull. Traumatol. Orthoped. N.N. Priorova, 1, 18-22.

Zakirova, E.Y., Vasin, N.N., Zhuravleva, M.N. and Rizvanov, A.A. 2014. Case report of application gene construction with VEGF and BMP2 in restoration of tear in the anterior cruciate ligament of a large breed dog. Genes Cells 9, 93-95. 